PEEHML 5 Nov, 1998

\title{
OPTIMAL EIGENVALUES FOR SOME LAPLACIANS AND SCHRÖDINGER OPERATORS DEPENDING ON CURVATURE
}

\author{
Pavel Exner* \\ Nuclear Physics Inst. \\ Academy of Sciences \\ 25068 Rez -Prague \\ Czech Republic \\ exner@ujf.cas.cz
}

\author{
Evans M. Harrell ${ }^{* *}$ and Michael Loss ${ }^{+}$ \\ School of Mathematics \\ Georgia Tech \\ Atlanta, GA 30332-0160, \\ USA \\ harrell@math.gatech.edu loss@math.gatech.edu
}

\begin{abstract}
We consider Laplace operators and Schrödinger operators with potentials containing curvature on certain regions of nontrivial topology, especially closed curves, annular domains, and shells. Dirichlet boundary conditions are imposed on any boundaries. Under suitable assumptions we prove that the fundamental eigenvalue is maximized when the geometry is round.

We also comment on the use of coordinate transformations for these operators and mention some open problems.

(C) 1998 by the authors. Reproduction of this article, in its entirety, by any means is permitted for non-commercial purposes.

* Work supported by GA AS No.1048801

** Work supported by N.S.F. grant DMS-9622730

+ Work supported by N.S.F. grant DMS-9500840
\end{abstract}




\section{Introduction}

In this article we present some theorems in optimal spectral geometry which were suggested by situations where the physics is strongly controlled by the geometry of an interface, i.e., a lower-dimensional structure. Two specific interests are electrical properties of nanoscale structures (quantum wires, waveguides, and resonators) [DuEx], and the slow evolution of interfaces in reaction-diffusion systems such as Allen-Cahn [AlFu].

By "optimal spectral geometry" we mean the determination of the geometry which maximizes or minimizes a certain eigenvalue of a differential operator. The archetypal result of this genre is the Faber-Krahn theorem [Fab] [Kra]:

1. If one considers the Dirichlet problem for the Laplacian on a region of specified volume (or area, in two dimensions), then it is the ball (disk) which uniquely minimizes the fundamental eigenvalue.

Other well-known theorems of optimal spectral geometry include:

2. (Weinberger [Wei]). If one considers the Neumann problem for the Laplacian on a region of specified volume, then the fundamental eigenvalue is trivially 0 . The first positive eigenvalue is uniquely maximized by the ball.

3. (Hersch [Her1]). If one considers the Laplacian on closed, simply connected surfaces of specified area embedded in $R^{3}$, then, as in the previous situation, the fundamental eigenvalue is trivially 0 . The first positive eigenvalue is then uniquely maximized by the sphere.

These theorems are sometimes termed "isoperimetric" in analogy with the classical isoperimetric theorem whereby the ball minimizes the surface area given a fixed volume. The simplicity of the optimizers in these situations may convey a misleading idea of the subtlety of the analysis involved. They involve more than simple rearrangement of the energy functional, and indeed the optimizers of higher eigenvalues are not so easy to characterize or even discover. Moreover, the theorem of Hersch is not true in all dimensions. For reviews of this subject, consult [AsBe], [Ban], and [Her2].

An exception to the statement about higher eigenvalues, however is the recent result in [HaLo], that the second eigenvalue of the Laplace operator penalized by the square of the mean curvature is uniquely maximized by the sphere. This result holds in any dimension. Interestingly, in two dimensions analogous facts can be proved, using Hersch's technique of conformal transplantation, for the second eigenvalue of the Laplace operator penalized by a substantially larger family of potentials quadratic in curvature (see [Har]).

In this article we shall present some new theorems where the fundamental eigenvalue is optimized by round geometry, and we attempt to shed light on the role of curvature in the spectra of Laplace and Schrödinger operators. In Section II, we consider the Laplacian on certain non-simply-connected domains and show that, under some circumstances, the optimization of the fundamental eigenvalue contrasts with the Faber-Krahn theorem. In Section III we review a transformation which has long been used to understand connections between curvature and spectra for quantum wires and waveguides, from the point of view of quadratic forms. This has consequences for further conjectures on spectral optimization and for the study of Schrödinger operators depending on curvature. In the final section we prove a new theorem on spectral optimization for some one-dimensional Schrödinger operators. 


\section{Isoperimetric spectral theorems for annuli and spherical shells}

In sections II-III we consider annular domains $D \subset R^{\nu+1}$, consisting of the points on one side of a closed, sufficiently smooth non-self-intersecting subset $\Omega$ of dimension $\nu$, and within a distance $d$ of $\Omega$. Our theorems will apply when $\nu=1$ or 2 , and where $d$ is sufficiently small, thus corresponding to physical structures such as quantum wires which form closed loops, or thin capacitors or resonating cavities with special geometries. We shall refer to $\Omega$ as the inner edge or the outer edge of the domain. The area (or length) of $\Omega$ will be written as $|\Omega|$.

The edge will be assumed sufficiently smooth that its principal curvatures are defined and bounded at all points (i.e., $\partial \Omega \in C^{2}$ ), and it is restricted so that all principal curvatures are bounded in magnitude by $1 / d$. (We choose the convention for plane curves which allows the curvature to have either sign. Later, when we treat surfaces, they will be assumed convex, so the principal curvatures will be positive.) This is an important constraint, which will be assumed throughout the article. In the language of differential geometry, it allows the existence of a Fermi coordinate system for $D$, consisting of a globally defined coordinate $r:=$ distance from $\Omega$, which is orthogonal to the coordinates on the smooth "level surfaces" $\Omega_{r}:=\{x \in D: \operatorname{dist}(x, \Omega)=r\}$. (We shall not make special assumptions about the coordinates on the level surfaces.) A set $D$ satisfying these assumptions will be called a smooth annular domain, and $d$ will be its thickness.

Theorem 1: a) With $\operatorname{dim}(\Omega)=1$, fix the length $|\Omega|$ and the thickness d,and consider the Dirichlet problem for the Laplacian on all smooth annular domains with $\Omega$ as one of the edges, either inner or outer. Then the fundamental eigenvalue $\lambda_{1}$ is uniquely maximized when $\Omega$ is a circle.

b) With $\operatorname{dim}(\Omega)=2$, fix the surface area $|\Omega|$ and the volume, and consider the Dirichlet problem for the Laplacian on all smooth annular domains $D$ with convex outer edges $\Omega$. Then the fundamental eigenvalue $\lambda_{1}$ is uniquely maximized when $\Omega$ is a sphere.

Remark. The assumptions in part a) are tantamount to fixing the area of the domain $D$, and thus when $\Omega$ is the outer edge statements a) and b) are analogous. The claim when $\Omega$ is the inner edge (or, by nearly the same proof, a central level curve) is an additional fact which appears to be valid only when $\Omega$ is one-dimensional.

Proof. a) We first consider the case $\nu=1$, and normalize so that $|\Omega|=2 \pi$. By the Rayleigh principle,

$$
\lambda_{1}=\inf \iint|\nabla \zeta|^{2} \mathrm{~d}^{2} x
$$

where the infimum is taken over smooth functions $\zeta$ on the closure of $D$ which vanish on its boundary, normalized in $L^{2}$. We write this in the orthogonal coordinate system defined by $r$ and $s:=$ arclength of $\Omega$ of the point nearest to $\mathbf{x}$, as measured counterclockwise from some reference position:

$$
\lambda_{1}=\inf \int_{0}^{d} \int_{0}^{2 \pi}\left(\frac{1}{(1 \pm \kappa(s) r)} \zeta_{s}^{2}+(1 \pm \kappa(s) r) \zeta_{r}^{2}\right) \mathrm{d} s \mathrm{~d} r
$$

(e.g., see $[\mathrm{Ban}]$, p. 143). Here, $\kappa$ is the curvature of $\Omega$ at $s$, and we have chosen the orientation whereby the plus sign corresponds to $\Omega$ being the inner edge, and the minus 
sign to it being the outer edge. Suppose now that a smooth test function $\zeta$ which vanishes on the boundary of $D$ is independent of $s$. Then

$$
\lambda_{1} \leq \int_{0}^{d} \int_{0}^{2 \pi}\left((1 \pm \kappa(s) r) \zeta_{r}^{2}\right) \mathrm{d} s \mathrm{~d} r=\int_{0}^{d}\left(2 \pi(1 \pm r) \zeta_{r}^{2}\right) \mathrm{d} r .
$$

The inequality would be strict if the true ground state were to depend on $s$. The final expression, however, is equivalent to the one for the corresponding annulus, restricted to the set of test functions independent of $s$. Since the fundamental eigenfunction for the circular annulus is attained in the set of functions independent of $s$, we conclude

$$
\lambda_{1} \leq \lambda_{1} \text { (annulus) }
$$

Because equality requires that the fundamental eigenfunction be independent of $s$, one of the two terms in the eigenvalue equation which includes $\kappa(s)$ is zero due to the presence of $\zeta_{s}$. This forces the only remaining term to be independent of $s$ for any curve $\Omega$ which maximizes $\lambda_{1}$. The only possibility is a circle.

b) Next we turn to the case $\nu=2$, and normalize so that $|\Omega|=4 \pi$, as for the unit sphere. We begin as before, by using the coordinate $r$, supplemented by coordinates orthogonal to $r$ on the level surfaces $\Omega_{r}$. It is convenient to denote $A(r):=\left|\Omega_{r}\right|$. Assuming that the test functions depend only on $r$, the Rayleigh principle states (for normalized test functions):

$$
\lambda_{1} \leq \int_{0}^{d} \zeta_{r}^{2} A(r) \mathrm{d} r
$$

We recall here that now the thickness $d$ depends on the domain $D$; specifically, they are connected by

$$
\int_{0}^{d} A(r) \mathrm{d} r=\operatorname{Vol}(D) .
$$

Let us now change variables to $r^{\prime}$ defined so that

$$
A_{0}\left(r^{\prime}\right) \mathrm{d} r^{\prime}=A(r) \mathrm{d} r
$$

where $A_{0}\left(r^{\prime}\right):=4 \pi\left(1-r^{\prime}\right)^{2}=$ the area of the sphere of radius $1-r^{\prime}$. We find:

$$
\lambda_{1} \leq \int_{0}^{d^{\prime}} \zeta_{r^{\prime}}^{2}\left(\frac{A(r)}{A_{0}\left(r^{\prime}\right)}\right)^{2} A_{0}\left(r^{\prime}\right) \mathrm{d} r^{\prime}
$$

where $d^{\prime}$ is the thickness of the spherical shell with the same volume as $D$. (For brevity we use informal notation for functions of transformed variables, etc.)

We next claim that the expression in parentheses is strictly smaller than 1 for $r>0$, unless $\Omega$ is a sphere. This is because the growth rate of the volume (here, area) element 
of the level surfaces is the sum of the principal curvatures (e.g., [Kar], eq. (1.5.4) or [Spi]. p. 418), and hence

$$
A^{\prime}(r)=-\int_{\Omega_{r}}\left(\kappa_{1}+\kappa_{2}\right) \mathrm{d} \sigma
$$

(cf. [Spi], p. 426). The negative sign arises here because $\Omega$ is the outer edge of the domain. At this stage we remark that if $\Omega$ is convex, then so are all the level surfaces in a smooth annular domain bounded by $\Omega$, and equation (2.1) can be integrated in a closed form discovered by Steiner ([San], p. 325):

$$
A(r)=A(0)-r \int_{\Omega_{0}}\left(\kappa_{1}+\kappa_{2}\right) d \sigma+4 \pi r^{2}
$$

Now recall a classical geometric lemma (e.g., [HiCo], p. 225):

Among all closed surfaces of fixed area, bounding a convex set, the sphere is the unique minimizer of the total mean curvature, $\int_{\Omega_{r}}\left(\kappa_{1}+\kappa_{2}\right) \mathrm{d} \sigma$.

We see that for a convex surface of a given area, the sphere is the unique maximizer of the negative quantity $A^{\prime}(r)$ for $r<d$. It then also follows that on this interval, $r^{\prime} \leq r$ and $0<A(r) \leq A_{0}(r) \leq A_{0}\left(r^{\prime}\right)$ with equality only in the case of the sphere.

The argument then proceeds as in part a): The upper bound obtained is equal to the Rayleigh quotient for the spherical shell, with radial test functions. Since the lowest eigenfunction for the spherical shell is radial, $\lambda_{1} \leq \lambda_{1}$ (shell), as claimed.

Theorem 1 contrasts with the Faber-Krahn theorem, which might lead one to expect that round annular domains were minimizers rather than maximizers. There are indeed other situations where $\lambda_{1}$ is maximized for the Laplace operator when the volume is fixed. One of these is addressed in a theorem of Payne and Weinberger [PaWe], on annular domains of a given area, where the outer edge is subject to Dirichlet conditions and the inner edge to Neumann conditions. (For some extensions of this theorem, see [Ban], section 4.3.)

Moreover, for the pure Dirichlet problem, the following is a special case of a theorem in $[\mathrm{HaKrKu}]$ :

Let the ball $B_{1}$ be contained inside the ball $B_{2}$. Fix the radii of $B_{1,2}$ but not the position of $B_{1}$ in $B_{2}$. Among all domains of the form $B_{2} \backslash B_{1}$, the one with the highest fundamental Dirichlet eigenvalue for the Laplacian is the one where they are concentric.

In these cases as well as in Theorem 1, the domain is allowed to vary within a class of non-simply connected domains. The contrast with Faber-Krahn thus seems to be a topological effect.

For reasons to be explored in the final section, we conjecture that the conclusion of the theorem is false if the dimension is increased to $\nu>2$.

\section{The natural coordinate transformation for the Dirichlet quadratic form}

In this section we begin with a coordinate transformation that is old and quite standard (e.g., [daC], $[\mathrm{DuEx}]$ ), using the Fermi coordinate system of the previous section. Our excuse for thus risking the reader's boredom is that we intend to use the transformation 
to illuminate an essential geometric feature of the effective potential-energy term which emerges. Also, we shall do the transformation in the context of quadratic forms rather than operators, which appears to us more direct and offers the possibility of relaxing some conditions of smoothness.

We continue to assume that our domain is a smooth annular domain with inner or outer edge $\Omega$. We construct a Fermi coordinate system using $r$, the distance from $\Omega$, as one coordinate, orthogonal to the rest. We denote the components of the gradient which are parallel to the level surfaces of constant $\mathrm{r}$ by $\nabla_{\|} f$.

We split the components of the Dirichlet form for the Laplace operator as:

$$
\int_{D}\left|\nabla_{\|} \zeta\right|^{2} \mathrm{~d}^{\nu+1} x+\int_{D}\left|\zeta_{r}\right|^{2} \mathrm{~d}^{\nu+1} x
$$

and transform the second term (only) as follows. Fix a coordinate system on the edge $\Omega$, and for any point $\mathbf{x}$ in $D$, choose as its coordinates other than $\mathrm{r}$ the coordinates of the closest point on the edge. Let $d V^{\nu}$ denote the volume element on $\Omega$. Then

$$
\int_{D}\left|\zeta_{r}\right|^{2} \mathrm{~d}^{\nu+1} x=\int_{\Omega} \int_{0}^{d}\left|\zeta_{r}\right|^{2} \rho(\mathbf{x}) \mathrm{dr} \mathrm{dV}^{\nu}
$$

where $\rho$ is the volume growth factor, a familiar quantity in differential geometry [Kar] [Spi]. We write the test function as

$$
\zeta=\frac{1}{\sqrt{\rho}} \cdot(\sqrt{\rho} \zeta)
$$

and use the product rule in the form

$$
\left((f g)^{\prime}\right)^{2}=f^{2}\left(g^{\prime}\right)^{2}+g^{2}\left(f^{\prime}\right)^{2}+\frac{1}{2}\left(f^{2}\right)^{\prime}\left(g^{2}\right)^{\prime}
$$

to find

$$
\int_{0}^{d}\left|\zeta_{r}\right|^{2} \rho \mathrm{dr}=\int_{0}^{\mathrm{d}}\left(\left|(\sqrt{\rho} \zeta)_{\mathrm{r}}\right|^{2}+\frac{1}{4}\left(\frac{\rho_{\mathrm{r}}}{\rho}\right)^{2} \zeta^{2} \rho+\frac{\rho}{2}\left(\frac{1}{\rho}\right)_{\mathrm{r}}\left(\rho \zeta^{2}\right)_{\mathrm{r}}\right) \mathrm{dr}
$$

When the final term is integrated by parts, the full Dirichlet form takes on the appearance

$$
\int_{A}\left|\nabla_{\|} \zeta\right|^{2} \mathrm{~d}^{\nu+1} x+\int_{A} q(\mathbf{x}) \zeta^{2} \mathrm{~d}^{\nu+1} \mathrm{x}+\int_{\Omega} \int_{0}^{\mathrm{d}}\left|(\sqrt{\rho} \zeta)_{\mathrm{r}}\right|^{2} \mathrm{dr} \mathrm{d} V^{\nu}
$$

The effective potential in the middle contribution is

$$
q(\mathbf{x}):=-\frac{1}{4}\left(\frac{\rho_{\mathrm{r}}}{\rho}\right)^{2}+\frac{1}{2} \frac{\rho_{\mathrm{rr}}}{\rho}
$$


In order to elucidate the geometric meaning of the effective potential, we recall two elementary facts about the geometry of level surfaces:

$$
\begin{gathered}
\text { (i) } \frac{\partial}{\partial r} \rho= \pm\left(\sum_{j=1}^{\nu} \kappa_{j}\right) \rho \\
\text { (ii) } \frac{\partial}{\partial r} \sum_{j=1}^{\nu} \kappa_{j}=\mp \sum_{j=1}^{\nu} \kappa_{j}^{2}
\end{gathered}
$$

Here, $\kappa_{j}$ are the principal curvatures of the level surface at coordinate $r$, and the choice of sign corresponds as usual to the choice of orientation. The signs of (i) and (ii) anticorrelate. (The two-dimensional version of (i) made an appearance in the previous section.)

A short calculation reveals an identity with unambiguous sign regardless of orientation:

Proposition 2: The Dirichlet form for the Laplacian is given by (3.1) with

$$
q(\mathbf{x})=\frac{1}{4}\left(\sum_{\mathrm{j}=1}^{\nu} \kappa_{\mathrm{j}}\right)^{2}-\frac{1}{2} \sum_{\mathrm{j}=1}^{\nu} \kappa_{\mathrm{j}}^{2} .
$$

Effective potentials of the form (3.2) have long been familiar in the study of microstructures with $\nu=1$ or 2 [daC] [DuEx] [ExŠe]. For the most part they have heretofore appeared as asymptotic calculations with the principal curvatures calculated on the edge, rather than on the level surfaces. In [DuEx] and [ExŠe] a variant effective potential throughout the domain is obtained by a "straightening" transformation. Roughly speaking, the latter articles replace the first term in (3.1) by an integral on a flat manifold, thus avoiding metric tensors, at the price of some derivatives of curvature in the potential energy.

We content ourselves here with a few simple observations about the uses of Proposition 2 , which we hope to explore further in a future article.

First, Proposition 2 is a tool for obtaining spectral bounds. A representative such bound is:

\section{Corollary 3:}

$$
\lambda_{1} \geq \frac{\pi^{2}}{\mathrm{~d}^{2}}+\inf (\mathrm{q}(\mathbf{x}))
$$

Proof. This follows immediately once it is realized that the final term in (3.1) can be considered as the Rayleigh quotient for the simple operator $-d^{2} / d x^{2}$ with Dirichlet boundary conditions at 0 and $d$, because the normalization is unchanged: $\|\sqrt{\rho} \zeta\|_{L^{2}\left(D, \mathrm{~d} r \mathrm{~d} V^{\nu}\right)}=$ $\|\zeta\|_{L^{2}\left(D, \mathrm{~d}^{\nu+1} x\right)} \cdot$

The first term of (3.1) is dropped and in the second the potential is replaced by its infimum.

Actually, sharper bounds than Corollary 3 are obtainable using analogues of the Boggio and Hardy inequalities [Bog] [Dav]. 
Next we observe that although the effective potential is nonpositive if $\nu=1(q(\mathbf{x})=$ $\left.-\frac{\kappa^{2}}{4}\right)$ or $2\left(q(\mathbf{x})=-\frac{\left(\kappa_{1}-\kappa_{2}\right)^{2}}{4}\right)$, as soon as $\nu>2$ the effective potential becomes positive for the sphere and many other hypersurfaces. While we have neither an isoperimetric theorem nor a counterexample to offer when $\nu>2$, the simplified model in the next section indicates that the situation can change dramatically when the effective potential may be positive.

\section{Spectral optimization for some Schrödinger operators depending on cur- vature}

In order to begin the analysis of the case where the effective potential is quadratic in the curvature but potentially positive, we consider the lowest eigenvalue of a family of one-dimensional operators parametrized by a real coupling constant $g$,

$$
H(g):=-\frac{d^{2}}{d s^{2}}+g \kappa^{2}
$$

We have thus simplified the situation of the previous section by reducing the dimension, but have allowed the effective potential to be positive when $g>0$. This model is also a natural generalization of one examined in [HaLo], corresponding to the case $g=-1$.

The operator $H(g)$ is defined on a closed planar curve normalized to have length 1 . If $g<0$, it is straightforward to see that of all curves, the circle maximizes the fundamental eigenvalue, as one might conjecture from Theorem 1 by considering infinitesimally thin annular domains approximating a closed curve. This would correspond only to $g=-1 / 4$, of course, but for all $g<0$ the statement is easy to obtain from the Rayleigh-Ritz inequality by considering the trial function $\zeta=1$ and making a simple estimate with the CauchySchwarz inequality.

The situation is more subtle when $g>0$.

Theorem 4: a) Suppose that $0<g<1 / 4$. Then the circle is the unique curve which minimizes the fundamental eigenvalue $\lambda_{1}$.

b) Suppose that $1<g$. Then the circle does not minimize the fundamental eigenvalue $\lambda_{1}$.

Remarks. The questions of the critical value of $g$ and the nature of the transition remain open. We conjecture that there is no legitimate minimizing curve when $g>1$.

Proof. a) Assume first that $0<g<1 / 4$. The minimal value of $\lambda_{1}$, which we denote $\lambda_{*}$, is

$$
\inf _{\kappa} \inf _{\zeta} \int\left(\left(\frac{\mathrm{d} \zeta}{\mathrm{d} s}\right)^{2}+g \kappa^{2} \zeta^{2}\right) \mathrm{d} s
$$

where the normalized $\zeta$ varies over the class of smooth periodic positive functions, while $\kappa$ is the curvature function defining a planar curve. The assumption of positivity may be imposed because of the positivity property of ground states of Schrödinger operators. We relax the conditions on $\kappa$ so that we require only that it be a function with integral $2 \pi$, i.e., we do not explicitly require that the curve be closed. We shall see that the minimizing curve is still a circle and therefore closed, so this causes no harm. 
Because the quantity in question is an iterated infimum, it may be calculated in the other order. By Cauchy-Schwarz's inequality

$$
2 \pi=\int \frac{\kappa}{\zeta} \zeta \mathrm{d} s \leq\left(\int \frac{1}{\zeta^{2}} \mathrm{~d} s\right)^{1 / 2}\left(\int \kappa^{2} \zeta^{2} \mathrm{~d} s\right)^{1 / 2},
$$

with equality only if

$$
\kappa=\left(2 \pi / \int \frac{1}{\zeta^{2}} \mathrm{~d} s\right) \frac{1}{\zeta^{2}} .
$$

Hence $\lambda_{*}$ is identical to the infimum of the unusual functional

$$
E(\zeta):=\int\left(\frac{\mathrm{d} \zeta}{\mathrm{d} s}\right)^{2} \mathrm{~d} s+\frac{4 \pi^{2} g}{\int\left(\frac{1}{\zeta^{2}}\right) \mathrm{d} s} .
$$

By choosing the trial function which becomes exact for the circle, i.e., $\zeta \equiv 1$, it follows that $\lambda_{*} \leq E(\zeta)=4 g \pi^{2}<\pi^{2}$ for $g<1 / 4$.

In order to establish the existence of a minimizer for $E(\zeta)$, we need a pointwise estimate:

Lemma 5: If $E(\zeta) \leq \pi^{2}$ for a positive test function $\zeta$ normalized in $L^{2}$, then

$$
\inf _{s}(\zeta(\mathrm{s}))>1-\frac{\sqrt{E(\zeta)}}{\pi} .
$$

Proof of Lemma 5.

$$
E(\zeta)>\int_{0}^{1}\left(\zeta^{\prime}\right)^{2} \mathrm{~d} s=\int_{0}^{1}\left(\zeta-\zeta_{\min }\right)^{\prime 2} \mathrm{~d} s \geq \pi^{2} \int_{0}^{1}\left(\zeta-\zeta_{\min }\right)^{2} \mathrm{~d} s,
$$

because $\zeta-\zeta_{\min }$ is an admissible test function for the operator $-d^{2} / d s^{2}$ with an additional Dirichlet boundary condition at the position of $\zeta_{\min }$, and that operator is bounded below by its lowest eigenvalue $\pi^{2}$, in the sense of quadratic forms. Hence

$$
E(\zeta)>\pi^{2}\left(\int_{0}^{1} \zeta^{2} \mathrm{~d} s-2 \zeta_{\min } \int_{0}^{1} \zeta \mathrm{ds}+\zeta_{\min }^{2}\right),
$$

which implies the claimed bound when we use the Cauchy-Schwarz inequality to replace $\int_{0}^{1} \zeta \mathrm{ds} \leq \sqrt{\int_{0}^{1} \zeta^{2} \mathrm{ds}}=1$ and then solve for $\zeta_{\min }$.

We now continue with the proof of Theorem 4. Because of the lemma, if $\lambda_{*}<\pi^{2}$, then any minimizing sequence for $E(\zeta)$ is bounded in the Sobolev space $H_{p e r}^{1}$. By a standard compactness theorem, a subsequence converges uniformly to a limit $\zeta_{*}$ which, by the lemma, is strictly positive. The same function is also a weak limit in the $H_{p e r}^{1}$ sense of a subsequence, from which it follows that $E\left(\zeta_{*}\right)=\lambda_{*}$ as claimed. 
We have thus established the existence of the minimizer $\zeta_{*}$, which is a nonnegative periodic function on the interval $[0,1]$. Next we observe that $\zeta_{*}$ satisfies the Euler equation for the functional $E$, which is found to be

$$
-\zeta_{*}^{\prime \prime}+M \frac{1}{\zeta_{*}^{3}}=C \zeta_{*},
$$

where

$$
M=\frac{4 \pi^{2} g}{\left(\int_{0}^{1} \frac{1}{\zeta_{*}^{2}} d s\right)^{2}}
$$

and $C$ is a Lagrange multiplier. Since $E\left(\zeta_{*}\right)=\lambda_{*}$, we find $C=\lambda^{*}$ by multiplying (4.1) by $\zeta_{*}$ and integrating by parts.

If the minimizer is a constant function, $\zeta_{*}(s)=1$, we have $\lambda_{*}=M=4 \pi^{2} g$. It remains to be seen whether $\zeta_{*}$ may be nonconstant. We multiply equation (4.1) by $\zeta_{*}^{\prime}$ and integrate; this yields

$$
\zeta_{*}{ }^{2}+\frac{M}{\zeta_{*}^{2}}+\lambda_{*} \zeta_{*}^{2}=C^{\prime}
$$

for some $C^{\prime}$. The minimizer is normalized by assumption, so integrating (4.2) we find $C^{\prime}=2 \lambda_{*}$. Hence the last equation can be rewritten as

$$
\zeta_{*}{ }^{2} \zeta_{*}{ }^{2}=\lambda_{*}-M-\lambda_{*}\left(\zeta_{*}{ }^{2}-1\right)^{2} .
$$

It follows that $\zeta_{*}$ oscillates between its (positive) minimal value $\sqrt{1-\sqrt{1-M / \lambda_{*}}}$ and the maximum $\sqrt{1+\sqrt{1-M / \lambda_{*}}}$, being strictly monotonic between them. The corresponding solution of (4.1) is given by

$$
\zeta_{*}^{2}=1+\sqrt{1-M / \lambda_{*}} \cos \left(2 \sqrt{\lambda_{*}}\left(s-s_{0}\right)\right) ;
$$

it is unique if we fix the point $s_{0}$ where the maximum is reached. However, a nonconstant function of this type cannot be periodic on [0,1], because $\lambda_{*}<\pi^{2}$.

b) Suppose now that $g>1$. The lowest eigenvalue for the circle is $4 \pi^{2} g$, so we need to show that lower eigenvalues are attainable, which we proceed to do with an explicit example. (While our example will have a discontinuous curvature, it can be approximated arbitrarily well by curves for which $\kappa(s)$ is perturbed arbitrarily slightly in the $L^{2}$ sense, which implies that the eigenvalues are shifted by arbitrarily small amounts [ReSi].) Consider the thin stadium-shaped curve defined by $\kappa(\mathrm{s})=\frac{\pi}{\varepsilon}$ for $1 / 2-\epsilon<s<1 / 2$ and $1-\epsilon<s<1$, and 0 otherwise, for some $\epsilon<<1 / 2$. Now estimate the fundamental eigenvalue using the Rayleigh-Ritz inequality and the trial function $\sin \left(\frac{\pi \mathrm{s}}{\frac{1}{2}-\varepsilon}\right)$ for $0<s<1 / 2-\epsilon$ and 0 otherwise; the result is $\lambda_{1} \leq\left(\frac{\pi}{\frac{1}{2}-\varepsilon}\right)^{2}$, which can be made arbitrarily close to $4 \pi^{2}$.

Remark: The proof of a) shows that the circle is also a minimizer if $g=1 / 4$, however we do not know whether it is the unique minimizer. 


\section{Acknowledgments}

The authors are grateful to Mark Ashbaugh, Michiel van den Berg, Rick Laugesen, and the referee for comments and references. E.H. wishes to thank the Erwin Schrödinger Institut and the Nuclear Physics Institute at Řž u Prahy for hospitality while some of this work was done.

\section{References}

[AlFu] Nicholas D. Alikakos and Giorgio Fusco, The spectrum of the Cahn-Hilliard operator for generic interface in higher space dimensions, Indiana U. Math. J. 4, 1993, pp. 637-674.

[AsBe] Mark S. Ashbaugh and Rafael D. Benguria, Isoperimetric inequalities for eigenvalue ratios, pp. 1-36 in: A. Alvino, E. Fabes, and G. Talenti, eds., Partial Differential Equations of Elliptic Type, Cortona, 1992. Cambridge: Cambridge University Press, 1994.

[Ban] Catherine Bandle, Isoperimetric inequalities and applications, Pitman Monographs and Studies in Mathematics 7. Boston: Pitman, 1980.

[Bog] Tommaso Boggio, Sull'equazione del moto vibratorio delle membrane elastiche, Rend. Accad. Lincei, sci. fis., ser. 5 16(1907)386-393.

[daC] R.C.T. da Costa, Quantum mechanics of a constrained particle, Phys Rev. A23(1981) 1982-1987 (and later articles).

[Dav] E.B. Davies, A review of Hardy inequalities, preprint 1998 available electronically as http://xxx.lanl.gov/abs/math.SP/9809159.

[DuEx] Pierre Duclos and Pavel Exner, Curvature-induced bound states in quantum waveguides in two and three dimensions, Rev. Math. Phys. 7(1995)73-102.

[ExŠe] Pavel Exner and Pavel Šeba, Bound states in curved quantum waveguides, J. Math. Phys. 30(1989)2574-2580.

[Fab] G. Faber, Beweis, dass unter allen homogenen Membranen von gleicher Fläche und gleicher Spannung die kreisförmige den tiefsten Grundton gibt, Sitzungsber. der mathematisch-physikalischen Klasse der Bayer. Akad. der Wiss. zu München (1923) $169-172$.

[Har] Evans M. Harrell II, On the second eigenvalue of the Laplace operator penalized by curvature, Journal of Differential Geometry and Applications 6(1996)397-400.

[HaKrKu] Evans M. Harrell II, Pawel Kröger, and Kazuhiro Kurata, work in progress.

[HaLo] Evans M. Harrell II and Michael Loss, On the Laplace operator penalized by mean curvature, Commun. Math. Physics 195(1998)643-650.

[Her1] Joseph Hersch, Quatre propriétés isopérimetriques de membranes sphériques homogènes, C.R. Acad. Sci. Paris, sér A-B 270, 1970, pp. A1645-1648.

[Her2] Joseph Hersch, Isoperimetric monotonicity: Some properties and conjectures (connections between isoperimetric inequalities), SIAM Review 30(1988)551-577.

[HiCo] D. Hilbert \& S. Cohn-Vossen. Geometry and the Imagination. New York: Chelsea, 1952.

[Kar] Hermann Karcher, Riemannian comparison constructions, pp. 170-222 in: S. Chern, editor, Global Differential Geometry, Studies in Mathematics 27. Washington: Math. Assoc. Amer., 1989. 
[Kra] E. Krahn, Über Minimaleigenschaften der Kugel in drei und mehr Dimensionen, Acta Comm. Univ. Tartu (Dorpat) A9(1926)1-44.

[PaWe] L.E. Payne and H.F. Weinberger, Some isoperimetric inequalities for membrane frequencies and torsional rigidity, J. Math. Anal. Appl. 2(1961)210-216.

[ReSi] M. Reed and B.Simon, Methods of Modern Mathematical Physics, IV: Analysis of Operators. New York: Academic Press, 1978.

[Spi] Michael Spivak, A Comprehensive Introduction to Differential Geometry, IV, second edition. Houston: Publish or Perish, 1979.

[San] L.A. Santalo, Integral Geometry, pp.303-350, in: S. Chern, ed., Global Differential Geometry, Studies in Mathematics 27. Washington: Math. Assoc. Amer., 1989.

[Wei] H.F. Weinberger, An isoperimetric inequality for the n-dimensional free membrane problem, J. Rat. Mech. Anal. 5(1956)633-636. 